and attempts to conceal the seizure (eg. by feigning a sneeze); no feeling of amusement accompanied the laughter. Of 5 patients with symptomatic, localization-related epilepsy, 4 had hypothalamic hamartoma $(\mathrm{HH})$ and 1 had tuberous sclerosis (TS). In 4 patients with cryptogenic gelastic seizures, the MRI was negative but seizures and EEG showed focal characteristics. Seizures were drug resistant in the $\mathrm{HH}$ cases, the TS patient responded to vigabatrin, and cryptogenic GS showed a variable outcome with partial seizure control. Two HH cases (ages 3 yrs and 28 yrs) showed complete or partial benefit following total surgical removal. Precocious puberty and cognitive deterioration, well recognized complications of the gelastic epilepsy-hypothalamic hamartoma syndrome of childhood, were not encountered in this series of patients. Cryptogenic cases of GS were usually more benign than the symptomatic HH cases. (Striano S, Meo R, Bilo L et al. Gelastic epilepsy: Symptomatic and crytogenic cases. Epilepsia March 1999;40:294-302). (Reprints: Prof Salvatore Striano, Viale dei Pini 101, 80131 Naples, Italy).

COMMENT. The childhood epileptic syndrome of early-onset gelastic seizures (GS; from the Greek 'gelos' meaning mirth), hypothalamic hamartoma $(\mathrm{HH})$, and precocious puberty (PP) is relatively rare but important in diagnosis and management. Frequent recurrent attacks of laughter, especially when accompanied by facial flushing, in a young infant or child may represent a recognized form of epilepsy and should prompt an MRI and EEG. The prognosis is usually poor, the GS evolving into symptomatic generalized epilepsy and leading to cognitive deterioration, but early recognition and surgical ablation of the hamartoma may occasionally result in control of drug refractory seizures and prevention of mental retardation.

The authors in this relatively large series of cases of gelastic epilepsy, including GS that are symptomatic of hamartomas and an equal number of cryptogenic cases, allude to the absence of precocious puberty, usually considered a classic sign of the syndrome, a later onset of seizures in some, and the relatively benign outcome of non-hamartoma cases.

Previous reports of gelastic epilepsy have usually involved only isolated cases. In 3 cases studied at Johns Hopkins University, GS originated in the anterior cingulate or fusiform gyri, and seizures were controlled following surgical removal of a cavernous hemangioma in one (Arroya S et al. Brain 1993;116:757780). Infantile spasms are often associated with attacks of paroxysmal laughter (Lacy JR, Penry JK. Infantile Spasms. New York, Raven Press, 1976). The classic epilepsy monographs of Turner WA (1907) and Lennox WG (1960;pp280-1) have references to compulsive laughter as a manifestation of epilepsy. Reference to GS is surprisingly absent from Penfield's monograph (1954). (See Progress in Pediatric Neurology II, Chicago, PNB Publishers, 1994;pp41-42, for reports and commentary on gelastic epilepsy).

\title{
LONG-TERM OUTCOME OF LANDAU-KLEFFNER SYNDROME
}

The types of language disorders in 4 young adults with Landau-Kleffner syndrome were analyzed at long-term follow-up at the National Institute of Mental Health, National Center of Neurology and Psychiatry, Chiba, Japan. Age of onset was 4 to 6 years, and the first symptoms of language disorder were an auditory verbal agnosia in 3 cases, and sensory aphasia in 1 . All patients showed a sequence of deterioration followed by recovery of language function without intellectual handicap. Sequelae included disability in spoken language, auditory verbal and music perception, and lower Wechsler Verbal scores compared to Performance IQ scores. Epilepsy began at 5 years in 2, and EEG epileptiform abnormalities occurred in all patients. CTs were normal in 3 and one showed an 
arachnoid cyst in the right anterior temporal lobe. (Kaga M. Language disorders in Landau-Kleffner syndrome. I Child Neurol Feb 1999;14:118-122). (Respond: Dr Makiko Kaga, Department of Developmental Disorders, National Institute of Mental Health, National Center of Neurology and Psychiatry, 1-7-3 Kohnodai, Ichikawa, Chiba 272, Japan).

COMMENT. Long-term follow-up of children with Landau-Kleffner syndrome into adult life shows a sequence of language disorders beginning at 4 to 6 years of age with sensory aphasia, and auditory verbal agnosia (word deafness), nonverbal agnosia, with or without anosognosia or denial of disability, and later showing improvement in auditory comprehension and speech but awareness of the impairments. The severity of language disorders, epileptic seizures, and EEG epileptiform discharges are not necessarily directly related, and antiepileptic medications which may control the seizures do not usually benefit the language deficits. Landau WM and Kleffner FR, in their original article (Syndrome of acquired aphsia with convulsive disorder in children. Neurology Aug 1957;7:523530), state that "EEG improvement tends to parallel improvement in speech reeducation."

Magnetoencephalography in Landau-Kleffner syndrome $h$ as been studied prior to subpial transection in 4 children in Helsinki, Finland. The earliest spike activity on the EEG originated in the intrasylvian cortex, and MEG data focussed on small otherwise overlooked EEG potentials, helping to identify the primary epileptogenic source and influencing the planning of surgery. ((Paetau R, Granstrom M-L. Blomstedt G et al. Magnetoencephgalography in presurgical evaluation of children with the Landau-Kleffner syndrome. Epilepsia March 1999;40:326-335).

\section{ANTIEPILEPTIC DRUGS}

\section{ENDOCRINE FUNCTION AND ANTIEPILEPTIC DRUGS}

The effects of valproate (VPA) in 40, carbamazepine (CBZ) in 19, and oxcarbazepine (OXC) in 18 girls with epilepsy on growth and maturation were compared to 49 healthy untreated controls examined at 8 to 18 years of age at the Departments of Neurology, Pediatrics, and Radiology, University of Oulu, Finland. VPA, CBZ, or OXC did not affect linear growth, followed longitudinally from the age of 1 year, or pubertal development. An increase in weight in VPA-treated girls occurred in those starting treatment either before or during puberty, and was not associated with hyperinsulinemia. High circulating concentrations of plasma insulin-like growth factor-1 in girls taking CBZ or OXC were of uncertain significance, and unassociated with abnormal weight gain. (Rattya J, Vainionpaa L, Knip M, Lanning P, Isojarvi JIT. The effects of valproate, carbamazepine, and oxcarbazepine on growth and sexual maturation in girls with epilepsy. Pediatrics March 1999;103:588-593). (Reprints: Johanna Rattya MD, Department of Neurology, University of Oulu, Kajaanintie 50, 90220 Oulu, Finland).

COMMENT. Neither VPA medication itself nor the associated weight gain affect linear growth or pubertal development of girls with epilepsy. Hyperinsulinemia, reported in obese adult women taking VPA for epilepsy, is not observed in girls treated for an average of 2.8 years. Weight gain in girls treated with VPA is slow and progressive and should be monitored regularly. These authors have reported on valproate-induced obesity and polycystic ovarian syndrome in women with epilepsy (see Progress in Pediatric Neurology III 1997). 